\title{
EDITORIAL Nanomedicine and cancer immunotherapy
}

Acta Pharmacologica Sinica (2020) 41:879-880; https://doi.org/10.1038/s41401020-0426-2

This special issue "Nanomedicine and Cancer Immunotherapy" was organized for the celebration of the 40th anniversary (1980-2020) of Acta Pharmacologica Sinica (APS). The prime aim for the special issue is to deliver both high-impact review and research articles to the wide international readership regarding the most recent progress in nano-immunotherapy as well as the approaches for enhancing the efficacy of cancer immunotherapy.

Primary tumors are conventionally treated by surgery, chemotherapy and radiotherapy. However, tumor relapse and ultimate therapeutic failure remained a formidable challenge in the clinic. The innate and adaptive immune system can make a substantial contribution to the therapeutic effects of conventional cancer treatments. Circumstantial evidence in pre-clinical studies suggests that the long-term success of cancer therapies lies in immunotherapy. Therefore, cancer immunotherapy is considered as an effective treatment modality to eliminate primary and metastatic tumors as well as to establish immunological memory. Nanomedicines can simultaneously deliver various immunological agents to the intended target site (tumor or lymph node). The ultimate application of nanomedicine is to reprogram or modulate the immune response by precisely targeting biological pathways. To increase cancer immunotherapies' impact, we organized this special issue to deepen our understanding about the mechanisms underlying the tumor and immune cells to evade immunity, how nanomedicines can be used to reprogram the tumor microenvironment and how nanomedicines interact with the immune system, and the potential challenges and the critical limitations of immunotherapy approaches, which impede their clinical applications. This issue includes one research article and ten review articles, and brief introductions to each topic are illustrated as below.

The advent of immunotherapy is a game changer in cancer therapy with monoclonal antibody and T-cell-based therapeutics being the current flagships. However, small molecule immunotherapeutics might offer advantages over biologicals in terms of complexity, tissue penetration, manufacturing cost, stability and shelf-life. However, small molecule drugs are prone to rapid systemic distribution which might induce severe off-target side effects. Nanotechnology could aid in the formulation of these drug molecules to improve their delivery to specific immune cell subsets. The review article by De Geest et al. summarizes the current efforts in changing the pharmacokinetic profile of small molecule immunotherapeutics with a strong focus on Toll like receptor agonists [1].

The review article by $\mathrm{Li}$ and $\mathrm{Nie}$ et al. introduces the strategies being employed using nanoscale intelligent drug delivery systems to enhance the effects of cancer immunotherapy by promoting drug delivery to the tumor. The review article also provides a perspective on the further possible application of nanoparticles in more effective anti-tumor immunotherapy [2].

Clinical approval of immune checkpoint inhibitors (e.g., Ipilimumab, Pembrolizumab) has been accelerated by a dramatic long-term survival improvement in a small subset of patients over conventional chemotherapy. However, it should be noted that the majority of treated cancer patients cannot benefit from checkpoint blockade therapies by releasing the T-cell function. Poor T-cell response, low immunogenicity and the immunosuppressive environment of tumors, create great challenges for present cancer immunotherapy. Nanotechnology can integrate multiple functions within controlled size and shape, and has been explored as a unique avenue for the development of cancer immunotherapy. The review article by Luo et al. mainly addresses how nanoengineered vaccine can induce robust $T$ cells response against tumor, as well as how nanomedicine can remodel immunosuppressive tumor microenvironment (ITM) to boost anti-tumor immune responses [3].

Advanced nanobiomaterials, including liposomes, polymers, and silica, play a vital role in the codelivery of drugs and immunomodulators. These nanobiomaterial-based delivery systems could effectively promote antitumor immune responses and simultaneously reduce toxic adverse effects. Furthermore, nanobiomaterials may also combine with each other or with traditional drugs via different mechanisms, thus giving rise to more accurate and efficient tumor treatments. The review article by Qian et al. provides an overview of the latest advancement of nanobiomaterials used for cancer immunotherapy including lipid- or micelle-based nanoparticles, polymer-based scaffolds, inorganic nanosystems, etc... [4].

A diversity of immunomodulatory agents, including tumorassociated antigens, adjuvants, cytokines and immunomodulators, have been explored for their ability to induce a cascading adaptive immune response. Nanoscale metal-organic frameworks (nMOFs) are attractive for cancer immunotherapy because they feature tunable pore size, high surface area and loading capacity, and intrinsic biodegradability. The review article by Sun et al. overviews the recent progresses in the development of nMOFs for cancer immunotherapy, including cancer vaccine delivery and combination of in situ vaccination with immunomodulators to reverse immune suppression. Current challenges and future perspectives for rational design of nMOF-based cancer immunotherapy are also discussed [5].

Central nervous system (CNS) disorders represent a broad spectrum of brain ailments with short and long-term disabilities. Despite the various nanomedicine-based platforms, translating therapeutic agents to the clinic is costly and often hampered by regulatory hurdles. A variety of potential drugs have been discovered to treat several neuronal disorders; however, therapeutic success is still limited by the presence of the blood-brain barrier (BBB). Furthermore, the unique immune mechanism of the CNS demands more consideration of immune-based therapeutic approaches for CNS diseases. The review article by Shi et al. discusses the efficacy of nanomedicines that utilize immunotherapy to combat CNS disorders for enhanced safety, efficacy, and drug delivery specificity [6].

Combining nanomedicines with immunotherapy aims to reinforce the cancer-immunity cycle, via potentiating key steps in the immune reaction cascade, namely antigen release, antigen processing, antigen presentation and immune cell-mediated tumor killing. Combination nano-immunotherapy can be realized via three targeting strategies, i.e. by targeting cancer cells, targeting the tumor immune microenvironment and targeting the peripheral immune system. The clinical potential of nano- 
immunotherapy has recently been demonstrated in a phase III trial in which nano-albumin paclitaxel (Abraxane ${ }^{\circledR}$ ) was combined with atezolizumab (Tecentriq ${ }^{\circledR}$ ) for the treatment of patients suffering from advanced triple-negative breast cancer. The review article by Shi and Lammers et al. summarizes the bedsides strategies and initial (pre)clinical success stories, and discusses several key challenges in nano-immunotherapy [7].

As the most powerful antigen-presenting cell type, dendritic cells (DCs) can induce potent antigen-specific immune responses in vivo, hence becoming the prime cell population for vaccination purposes. DCs can be derived ex vivo in large quantity and manipulated extensively to be endowed with adequate immunestimulating capacity. After pulsing with cancer antigens in various ways, the matured DCs are administered back into the patient. DCs home to lymphoid organs to present antigens to and activate specific lymphocytes that react to a given cancer. Ex vivo pulsed DC vaccines have been vigorously investigated for decades, registering encouraging results in relevant immunotherapeutic clinical trials, while facing some solid challenges as well. The review article by Song et al. reports on the advances of DC vaccine-based cell immunotherapy, in particular the application of mRNA technology for the fabrication of DC vaccines [8].

Cancer immunotherapy has attracted extensive attention due to its ability to activate the patients' innate or adaptive immune systems to combat tumors. Despite a few clinical successes, further endeavors are still needed to tackle unresolved issues including a limited response rate, development of acquired immune resistance and immune-related toxicities. Accumulating evidence has pinpointed tumor microenvironment (TME) as one of the major obstacles against cancer immunotherapy due to its detrimental impacts on tumor-infiltrating immune cells. Nanomedicine has been battling with the TME in the past decades and experience obtained could be exploited to improve the current paradigms of immunotherapy. The review article by Wang et al. discusses the metabolic features of TME and its influence on different types of immune cells, and summarizes the recent progress of nano-enabled cancer immunotherapy by modulation of immune cells, tumor stroma, cytokines and enzymes to revert immunosuppressive TME [9].

Over the past years, several treatment modalities including radiotherapy, chemotherapy, photothermal and photodynamic therapy, have been exploited to elicit immunogenicity by inducing immunogenic cell death (ICD). However, ICD-based immunotherapy is restricted by the ITM limiting its efficacy in eliciting a long-term anti-tumor immune response as well as severe systemic toxicity. To address these challenges, nanomedicine-based drug delivery strategies have been exploited to improve cancer immunotherapy by boosting ICD of tumor cells. Nanosized drug delivery systems are promising for increasing drug accumulation at the tumor site and co-delivering ICD inducers and immune inhibitors to simultaneously elicit the immune response and relieve the ITM. The review article by $\mathrm{Yu}$ et al. highlights the recent advances in nanomedicine-based immunotherapy utilizing ICD-based approaches [10].

The research article by Rock et al. reports Enzyme-Directed Immunostimulant (EDI) prodrugs for cancer immunotherapy. The tested EDIs feature an imidazoquinoline immunostimulant (resiquimod-Toll-Like Receptor 7/8 agonist) covalently modified with glycosidase enzyme-directing groups selected from substrates for $\beta$-glucuronidase, a-mannosidase, or $\beta$-galactosidase. The elicited immunogenicity was compared for different cell types and found to be independent of the glycosidase substrate in the EDI or differences in functional glycosidase expression across each cell line. This study highlights the broad applicability of EDls to the cancers that exhibit drug efflux, which suggest that EDIs optimized for drug efflux could be particularly beneficial for improving cancer immunotherapy [11].

We would like to express our sincere gratitude to all the eminent contributors, who have made such significant contributions to this special issue in Acta Pharmacologica Sinica, and would also like to extend our gratitude to all the peer-reviewers for their insights and thoughtful comments, which significantly improved the quality of this issue. Meanwhile, we want to express our deep gratitude to Professor Jian Ding, the Editor-in-Chief, and Mrs. Ming-shu Wu, Manager-in-Chief of Acta Pharmacologica Sinica, for their invitation and kindly help throughout the whole process. Without their initiative and encouragement, this special issue would not have been possible. Last but not least, we must greatly acknowledge Ms. Juan Huang and Mr. Jia Xu, the production editors of Acta Pharmacologica Sinica, for their remarkable work done for this special issue. The last but not the least, it is our hope that all of the articles in this special issue will be found helpful and useful to both established and new investigators in the field of Nanomedicine and Cancer Immunotherapy.

\section{Competing interests}

The authors declare no competing interests.

Hai-jun $\mathrm{Yu}^{1}$ and Bruno $\mathrm{G}$ De Geest ${ }^{2}$ ${ }^{1}$ State Key Laboratory of Drug Research \& Center of Pharmaceutics, Shanghai Institute of Materia Medica, Chinese Academy of Sciences, Shanghai 201203, China and ${ }^{2}$ Department of Pharmaceutics, Ghent University, Ghent, Belgium Correspondence: Hai-jun Yu (hjyu@simm.ac.cn) or Bruno G De Geest (br.degeest@ugent.be)

\section{REFERENCES}

1. Van Herck S, De Geest BG. Nanomedicine-mediated altering of the pharmacokinetic profile of small molecule cancer immunotherapeutics. Acta Pharmacol Sin. 2020;41:881-94.

2. Qi FL, Wang MF, Li BZ, Lu ZF, Nie GJ, Li SP. Reversal of the immunosuppressive tumor microenvironment by nanoparticle-based activation of immune-associated cells. Acta Pharmacol Sin. 2020;41:895-901.

3. Yin WM, Li YW, Gu YQ, Luo M. Nanoengineered targeting strategy for cancer immunotherapy. Acta Pharmacol Sin. 2020;41:902-10.

4. Yang F, Shi K, Jia YP, Hao Y, Peng JR, Qian ZY. Advanced biomaterials for cancer immunotherapy. Acta Pharmacol Sin. 2020;41:911-27.

5. Zhong XF, Sun X. Nanomedicines based on nanoscale metal-organic frameworks for cancer immunotherapy. Acta Pharmacol Sin. 2020;41:928-35.

6. Hanif $S$, Muhammad $P$, Chesworth R, Rehman FUR, Qian RJ, Zheng $M$, et al. Nanomedicine-based immunotherapy for central nervous system disorders. Acta Pharmacol Sin. 2020;41:936-53.

7. Sun QX, Bai XY, Sofias AM, Meel R, Ruiz-Hernandez E, Storm G, et al. Cancer nanomedicine meets immunotherapy: opportunities and challenges. Acta Pharmacol Sin. 2020;41:954-8.

8. Gu YZ, Zhao X, Song XR. Ex vivo pulsed dendritic cell vaccination against cancer. Acta Pharmacol Sin. 2020;41:959-69.

9. Sun B, Hyun H, Li LT, Wang AZ. Harnessing nanomedicine to overcome immunosuppressive tumor microenvironment. Acta Pharmacol Sin. 2020;41:970-85.

10. Gao J, Wang WQ, Pei Q, Lord MS, Yu HJ. Engineering nanomedicines for improved cancer immunotherapy by boosting immunogenetic cell death. Acta Pharmacol Sin. 2020;41:986-94.

11. Ryan AT, Pulukuri AJ, Davaritouchaee $M$, Abbasi A, Hendricksen AT, Opp LK, et al. Comparing the immunogenicity of glycosidase-directed resiquimod prodrugs mediated by cancer cell metabolism. Acta Pharmacol Sin. 2020;41:995-1004. 